\title{
Financiamiento de programas de farmacodependencia en la ciudad de México: 1990-1994
}

Irene M. Parada-Toro, M.C., M. en C., (1) Patricia H ernández-Peña, M.C., Ph.D., ${ }^{(2)}$ Armando Arredondo-López, M.C., M. en C., Ph.D., ${ }^{(1)}$ Francisco Becerra-Posada, M.C., M. en S.P. ${ }^{(3)}$

\begin{abstract}
Parada-Toro IM, Hernández-Peña $P$, Arredondo-López A, Becerra-Posada F. Financiamiento de programas de farmacodependencia en la ciudad de México: 1990-1994. Salud Publica Mex 2000;42:118-125.
\end{abstract}

\section{Resumen}

Objetivo. Analizar el flujo del financiamiento de los programas de farmacodependencia en la ciudad de México, sus fuentes y asignaciones. Material y métodos Se seleccionó un grupo discreto de instituciones dedicadas a la prevención y el control de la farmacodependencia en la ciudad de México, incluyendo a las instituciones públicas más importantes en el ramo. Se aplicaron encuestas entre administradores y usuarios. Además de integrar los flujos financieros se determinó el gasto por usuario de cada institución. Para el periodo 1990-1993, los gastos se estimaron a partir de los montos financieros asignados a cada programa en 1990 y a valores constantes, para eliminar el efecto de la inflación. Resultados Las fuentes de financiamiento de las instituciones del grupo estudiado son diversas: de 50 a $90 \%$ provienen del presupuesto federal; de 10 a 20\%, de aportaciones de los usuarios, y de 15 a 80\%, de contribuciones de organizaciones no gubernamentales. Conclusiones.A unque se han incrementado los montos financieros, en los últimos cuatro años, son insuficientes para el desarrollo de los programas en las instituciones de este estudio, ante la creciente demanda. Se requiere establecer mecanismos que aseguren la captación de recursos necesarios y su uso eficiente. El análisis financiero continuo de estos programas permitirá la toma de decisiones oportuna y una evaluación de los cambios.

Palabras clave: financiamiento de la salud/dependencia de sustancias; México

\author{
Parada-Toro IM, Hernández-Peña $P$, \\ Arredondo-López A, Becerra-Posada F. \\ Financing of drug \\ dependency programs \\ in Mexico City: 1990-1994. \\ Salud Publica Mex 2000;42:118-125.
}

\begin{abstract}
A bstract
Objective. To analyze the financial flows in the drug dependency programs in México City, their financial source, and the way how funding monies are spent. Material and methods. A discrete number of institutions devoted to drug dependency control in Mexico City was selected. Analysis of financial flows was carried out using the $\mathrm{N}$ ational $\mathrm{H}$ ealth Accounts methods. Q uestionnaires to quantify resources were applied to health service managers and users. In addition to collection of financial flow data, we calculated the expenditures per user for each institution. For the period 1990-1993, expenditures were estimated from financial budgets allocated to each program in 1990, at constant values to eliminate the effects of inflation. Results. Financial sources identified were: the federal budget allocation (50-90\%), user fees (10-20\%), and non-governmental organization contributions (15-80\%). Conclusions. Even though budgets in participant institutions have increased in the last four years, they are still inadequate to cover the large demand of services. It is important to identify new ways to increase the availability of resources as well as to ensure their efficient use. Continuing financial analysis of these programs will allow the assessment of changes to guide optimal financial decision making.
\end{abstract}

Key words: financing, health/substance dependence; Mexico

(1) Centro de Investigación en Sistemas de Salud, Instituto Nacional de Salud Pública, México.

(2) Fundación Mexicana para la Salud, México.

(3) Dirección de Desarrollo y Administración de Redes, Meximed, México.

Fecha de recibido: 23 de julio de 1999 • Fecha de aprobado: 20 de enero de 2000

Solicitud de sobretiros: Dra. Irene M. Parada-Toro. Centro de Investigación en Sistemas de Salud, Instituto N acional de Salud Pública. A venida Universidad 655, 2do. Piso, colonia Santa María A huacatitlán, 62508 Cuernavaca, Morelos, México.

Correo electrónico: mparada@insp3.insp.mx 
L a atención a la farmacodependencia se ha convertido en un problema importante de salud pública para los sistemas de salud en México, aunque la forma en que se enfrenta es poco conocida. ${ }^{1,2}$ Además, se carece de información sobre el financiamiento de los programas destinados a la rehabilitación de farmacodependientes. Ante el vacío documental existente, en este trabajo se presentan los resultados de un estudio sobre las modalidades, las fuentes y los flujos financieros de los programas de atención a la farmacodependencia. ${ }^{3,4}$

La farmacodependencia es un complejo mecanismo mediante el cual los individuos intentan compensar carencias, incapacidades y necesidades consumiendo drogas. De hecho, su estudio no sólo considera el aspecto de la salud mental, sino también las características culturales y sociales del entorno actual. De ello se deduce que la farmacodependencia es un síntoma o indicador de las incongruencias, deficiencias o limitaciones de las estructuras sociales de un país. ${ }^{5}$

La formalización de toda política y los programas correspondientes deben asegurar su viabilidad por medio de la suficiencia y la oportunidad de sus recursos financieros. La planeación financiera actualmente debe operar sistemas flexibles de movilización de recursos que aseguren la sustentabilidad de los programas. De ahí la importancia de conocer los flujos financieros en los servicios de atención a la farmacodependencia, de tal manera que se pueda hacer un diagnóstico al respecto y se establezca un punto de referencia para operar los cambios en el financiamiento que se requieran.

En la literatura revisada sobre farmacodependencia no hay antecedentes acerca del tipo, la estructura y el monto del financiamiento, ni del papel de las organizaciones no gubernamentales (ONG) que intervienen en la atención a farmacodependientes.$^{6-8}$ Estudios anteriores reconocen como prioridad atender la prevención de la farmacodependencia por ser costo-efectiva, ${ }^{9}$ pues el tratamiento de un paciente inserto ya en el ambiente de las drogas requiere de una intervención de alto costo.

En México, desde 1994, existen estudios para el análisis de los flujos financieros en los servicios de salud, pero no han llegado a detallar los de programas específicos como el de la atención a la farmacodependencia. ${ }^{10-12}$

Por ello, y como un primer abordaje de este tema, el presente estudio de caso analiza los flujos financieros, es decir, las diferentes instancias por las que pasan los recursos monetarios, hasta llegar a su destino final, en instituciones con programas dirigidos a atender la farmacodependencia en la ciudad de México.

\section{Material y métodos}

Se realizó un estudio de caso. Se analizaron cuatro (27\%) de las 15 instituciones que hasta 1993 estaban registradas en el Consejo Nacional para las Adicciones (Conadic). Las instituciones estudiadas están ubicadas en la ciudad de México; dos de ellas atienden a población no asegurada, una está constituida como asociación civil y la otra es privada.

La información corresponde al periodo 1990-1993, el estudio se realizó entre 1993 y 1994, a partir de dos encuestas: una dirigida a los administradores $y$, otra, a los usuarios de las instituciones seleccionadas para el estudio. Los datos se complementaron con la revisión de fuentes secundarias como los registros financieros, las características institucionales y los programas.

Los criterios de selección de las instituciones fueron:

- Formar parte del directorio del Consejo Nacional para las Adicciones.

- Cubrir a la población mayor de ocho años.

- Estar localizadas en la ciudad de México.

- Prestar servicios de psiquiatría, psicología, de prevención y tratamiento de la farmacodependencia, así como de rehabilitación, mediante servicios ambulatorios u hospitalización.

Para guardar la confidencialidad, a cada institución se le asignó un número:

1. Institución privada (con fines de lucro).

2. Asociación civil (privada sin fines de lucro).

3. Institución pública dedicada atender a población no asegurada.

4. Institución pública dedicada a atender a población no asegurada infantil.

Las variables analizadas fueron: precio de la consulta, cobertura de la institución, contenido de los programas y financiamiento. La información se organizó para cubrir las siguientes categorías: ${ }^{13}$

1. Descripción del flujo financiero.

2. Identificación de las fuentes de financiamiento y cuantificación de las aportaciones que hacen las mismas.

3. Identificación de los fondos financieros y de su asignación según tipo de programa en cada institución.

4. Descripción de los precios de la consulta y determinación de la cobertura institucional. 
Además de integrar los flujos financieros se determinó el gasto por usuario de cada institución.*

\section{Resultados}

Para el periodo 1990-1993, los gastos se estimaron a partir de los montos financieros asignados a cada programa en 1990 y a valores constantes, para eliminar el efecto de la inflación.

La respuesta de las instituciones para el análisis de la dinámica financiera de la atención a la farmacodependencia fue poco entusiasta. Sin embargo, el grupo de instituciones que aceptó participar en el estudio permitió obtener una panorámica de sus flujos financieros y de su composición heterogénea (figura 1).

\section{Recursos disponibles}

El monto de recursos disponibles por cada institución tuvo una dinámica de crecimiento irregular durante los cuatro años de estudio y mostró un discreto in-

* Fuente: Banco de México. Indice de precios, 1990-1991. cremento a valores constantes. Comparativamente, la institución 1 (privada con fines de lucro) contaba con el mayor presupuesto, el cual era casi tres veces más grande que el de la número 2, 10 veces más amplio que el de la número 3 y 74 veces mayor que el de la número 4 . La institución 1 mantuvo un presupuesto relativamente estable en el tiempo a precios constantes de 1994, excepto en el último año, cuando lo duplicó; la institución 2 (privada sin fines de lucro), en cambio, durante el periodo presentó un incremento presupuestal que casi sextuplicó el monto de 1990 a valores constantes. Por su parte, las instituciones 3 y 4 incrementaron su presupuesto cuatro veces a lo largo del periodo (cuadro I).

\section{Fuentes de financiamiento}

Las instituciones estudiadas obtienen recursos de distintas fuentes de financiamiento: empresas (73\%), hogares $(23 \%)$ y gobierno $(3 \%)$, así como organismos internacionales. Estos últimos no fueron cuantificados, pero se reconocieron como importantes para una de las instituciones estudiadas.

Mientras que en las instituciones públicas se evidencia una mezcla público-privada en su financia-

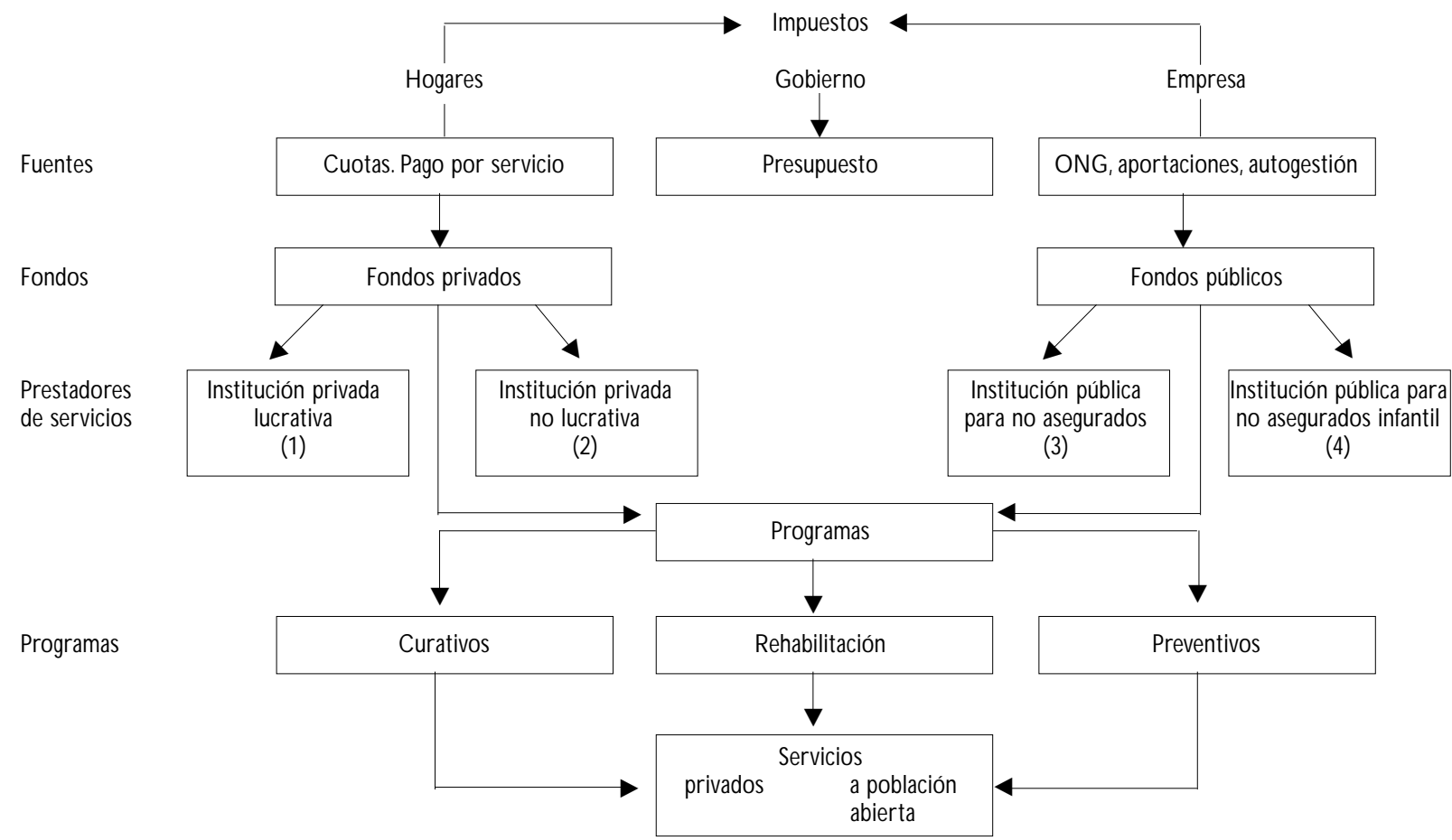

Figura 1. Flujos financieros. Instituciones de atención a la farmacodependencia. México, 1990-1993 
Cuadro I

Presupuesto anual por institución a pesos CONSTANTES Y CORRIENTES. INSTITUCIONES DE ATENCIÓN A LA FARMACODEPENDENCIA. MÉXICO, 1990-1993

\begin{tabular}{lccc} 
Instituciones & Año & Pesos constantes & Pesos corrientes \\
$N 0.1$ & 1990 & 2295.0 & 146880.0 \\
\hline $\mathrm{N} 0.1$ & 1991 & 2895.0 & 156348.0 \\
\hline $\mathrm{N} 0.1$ & 1992 & 3660.0 & 146400.0 \\
\hline $\mathrm{N} 0.1$ & 1993 & 5310.0 & 159840.0 \\
\hline $\mathrm{N} 0.2$ & 1990 & 301.47 & 19294.4 \\
\hline $\mathrm{N} 0.2$ & 1991 & 529.67 & 28602.4 \\
\hline $\mathrm{N} 0.2$ & 1992 & 1244.26 & 49770.6 \\
\hline $\mathrm{N} 0.2$ & 1993 & 1880.62 & 56606.9 \\
\hline $\mathrm{N} 0.3$ & 1990 & 121.44 & 7772.5 \\
\hline $\mathrm{N} 0.3$ & 1991 & 192.41 & 10390.2 \\
\hline $\mathrm{N} 0.3$ & 1992 & 314.42 & 12577.0 \\
\hline $\mathrm{N} 0.3$ & 1993 & 517.89 & 15588.5 \\
\hline $\mathrm{N} 0.4$ & 1990 & 15.79 & 1010.7 \\
\hline $\mathrm{N} 0.4$ & 1991 & 22.60 & 1220.5 \\
\hline $\mathrm{N} 0.4$ & 1992 & 41.87 & 1672.7 \\
\hline $\mathrm{N} 0.4$ & 1993 & 71.57 & 2154.4
\end{tabular}

Las deflactaciones se realizaron con base en el informe del Banco de México para índices de precios 1994=100

miento, en la institución 1 los recursos son totalmente privados, pues la mayor parte $(80 \%)$ proviene de las cuotas de recuperación, y la proporción restante $(20 \%)$ es provista por una ONG nacional (empresa sin fines de lucro). Los recursos de la institución 2 son también principalmente privados, aunque provienen de una mayor diversidad de fuentes: recibe donaciones de una ONG nacional $(10 \%)$, del patrimonio de la beneficencia pública $(15 \%)$, y la mayor parte de sus recursos provienen de la autogestión (75\%). Esta institución (la número 2) recibe también donaciones en especie de la Central de Abastos. Sus recursos de autogestión se generan a partir de la operación de dos empresas propias de venta de alimentos.

Las instituciones 3 y 4 son públicas, pues su principal fuente financiera es el gobierno en 50 y $90 \%$ de sus ingresos, respectivamente; el resto de sus recursos es privado, pues proviene de las cuotas de recuperación que aportan los usuarios. No hay referencia de prepagos en ninguna de las instituciones estudiadas; en el caso de la institución 1, los usuarios realizan pagos de bolsillo al utilizar el servicio, y en la institución 2 el servicio es gratuito, por lo que los aportes adicionales corresponden a pequeñas donaciones de los familiares de los pacientes. En las instituciones 3 y 4 , el monto de las cuotas de recuperación que reciben depende de un estudio socioeconómico que se realiza entre los usuarios al momento de recibir el servicio. Los recursos privados mayores provienen de los hogares como pago de servicios; le siguen la autogestión y las donaciones de las ONG. Las cuotas de recuperación en instituciones públicas corresponden a los menores aportes (figura 2).

De 1990 a 1993, la institución 3 dedicó 67\% de su presupuesto a la prevención (rubro que incrementó su presupuesto casi al doble en el periodo, alcanzando $71 \%$ en 1993) y $33 \%$ a consulta externa, actividad en la que se redujo el gasto. Es preciso observar que de las cuatro instituciones estudiadas, ésta es la que destina mayor presupuesto a la prevención; quizá ello se deba a que es la institución con mayor apoyo estatal y cobertura en el país para el tratamiento de la farmacodependencia. Además, esta institución depende de un monto reducido de recursos totales y los distribuye diversificadamente según el tipo, y número de usuarios y el tipo de acciones.

En general, el monto de los recursos es muy diferente en las instituciones, pues muestra una asignación per cápita que varía hasta 200 veces en los casos extremos. Llama la atención que la institución 4 , que otorga servicios a la población infantil no asegurada, dispone de la menor proporción de recursos (cuadro II).

Las coberturas de las instituciones visitadas difieren en tamaño. Todas reflejan una trayectoria heterogénea: la número 1 presentó, en cuatro años, un

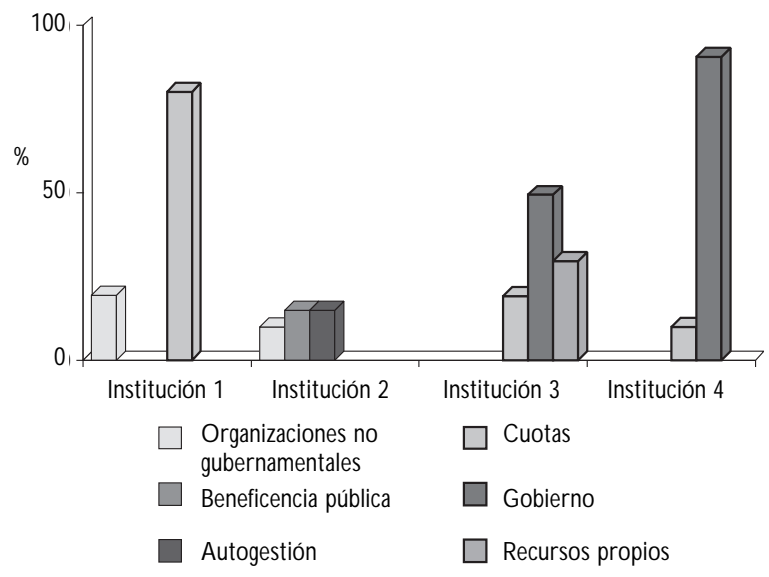

Figura 2. Fuentes financieras institucionales. INSTITUCIONES DE ATENCIÓN A LA FARMACODEPENDENCIA. Méxıco, 1990-1993 


\section{Cuadro II \\ MONTO del GASTO POR USUARIO EN CADA INSTITUCIÓN, EN PESOS DE 1993. INSTITUCIONES DE ATENCIÓN A LA FARMACODEPENDENCIA. MÉXICO, 1990-1993}

$\begin{array}{lc}\text { Institución } & \text { Monto per cápita } \\ \begin{array}{lc}\mathrm{N} 0.1 & 532.8 \\ \hline \mathrm{N} 0.2 & 235 \\ \hline \mathrm{N} 0.3 & 3.86 \\ \hline \mathrm{N} 0.4 & 2.56 \\ \hline \text { Media per cápita* } & 43.26 \\ \text { * Media del monto de } 1993 & \end{array}\end{array}$

aumento de 50\% en su cobertura (de 200 casos tratados en 1990, a 300 para 1994), y su gasto incrementó en $9 \%$; la cobertura de la número 3 , en cambio, decreció en 35\% (de 366335 casos tratados en 1990, a 272666 en 1994), y sus recursos aumentaron $100 \%$. Esta última institución tenía un gasto por persona muy reducido.

La institución privada número 1 presenta un aumento constante en la cobertura, mientras que durante los años 1990-1993, la institución 3 tuvo un decremento significativo en su cobertura real. Sin embargo, los datos indican que la institución con mayor cobertura potencial es la número 3 , porque atiende a la población no asegurada que representa $15.9 \%$ de la población farmacodependiente registrada en la Encuesta Nacional de Adicciones de 1988 y 1994 y que cuenta con las características socioeconómicas para ser atendida por esta institución. ${ }^{14,15}$
Las cuotas que los usuarios aportan a las instituciones dedicadas a cubrir la demanda de la población no asegurada son reducidas y no cubren los costos generados por el tratamiento. El valor de estas cuotas oscila entre 1 y 20 pesos (0.30-6.06 dólares). Este precio está muy por debajo del pagado por la población que recibe tratamiento en las instituciones privadas, pues es de 10 a 22 veces mayor. Aunado a ello, del total de la población estudiada en las instituciones 3 y 4 , sólo $43 \%$ paga por los servicios, y en $99 \%$ de los casos, las cuotas son mínimas, ya que van de 1 a 5 pesos.

El monto del gasto per cápita demuestra que los usuarios que reciben más recursos son los que acuden a las instituciones 1 y 2 , es decir, a aquellas dedicadas a cubrir a la población que busca atención privada, no así a las que reciben servicios para población no asegurada (cuadro II).

\section{Programas}

Las instituciones estudiadas realizan diferentes actividades para el tratamiento de la farmacodependencia. Es importante señalar que este tipo de atención, debido al carácter complejo del padecimiento que trata, necesita, por un lado, de un trabajo individualizado con el paciente y, por el otro, con la familia, además de que debe lograr la reinserción del individuo en la sociedad. Por este motivo, se utiliza una gran variedad de técnicas en el tratamiento y la rehabilitación, (cuadro III). En dos de las instituciones se realizan actividades preventivas $y$, en las restantes, acciones de rehabilitación. En todas se llevan a cabo tratamientos

\section{Cuadro III \\ Programas brindados por cuatro instituciones de atención A la farmacodependencia. MÉxico, 1990-1993}

Institución Programas

No.1 Clínico, o de prevención y rehabilitación. Hospitalización durante los tres meses de tratamiento, y atención ambulatoria después del alta

No.2 Clínico y de rehabilitación. Hospitalización durante tres meses

N $0.3 \quad$ Clínico (ambulatorio) y preventivo

No.4 Clínico (ambulatorio) y preventivo
Definición del tratamiento

Clínico: tratamiento psiquiátrico y psicológico, este último en sus vertientes de terapia individual, familiar y de grupo. La rehabilitación se lleva cabo con talleres de lectura y terapia ocupacional, entre otras actividades.

Clínico: atención psiquiátrica para desintoxicación y terapias similares a las de Alcohólicos A nónimos. La rehabilitación consiste en terapias ocupacionales.

Psiquiátrico y psicológico, este último en sus vertientes de terapia individual y familiar. Se realiza labor preventiva en los ámbitos comunitario y escolar.

Asistencia psiquiátrica en consulta externa y atención psicológica en su vertiente de terapia grupal. El tratamiento preventivo sólo se realiza en el nivel escolar primario. 
clínicos; sin embargo, el enfoque de las actividades terapéuticas es diverso (cuadro III).

La atención preventiva representa $71 \%$ del presupuesto de la institución 3, y de la atención clínica, la ambulatoria representa $100 \%$ del gasto de la institución 4 y casi 29\% de la número 3. En la institución 2 la atención hospitalaria se brinda con un gasto de $37 \%$ de su presupuesto. La rehabilitación se realiza en la institución 1 y en la institución 2, con 63\% del presupuesto.

En conjunto, la atención preventiva representa $8 \%$ del gasto en las instituciones estudiadas; la atención clínica, 68\% del gasto, dividido entre el que se destina a la atención ambulatoria (53\%) y el que se asigna a la hospitalaria (15\%); la rehabilitación se lleva $24 \%$.

\section{Discusión}

Independientemente del tipo de institución de que se trate, no es posible acceder a sus indicadores financieros. Esto pone de manifiesto la ausencia de una cultura de análisis financiero y de un funcionamiento transparente en este campo, propio de la época en que se realizó el estudio.

Llama la atención la heterogeneidad de la dinámica financiera en las cuatro instituciones de estudio, así como sus efectos en el gasto per cápita. El gasto promedio por paciente fue de 43 pesos de 1994, equivalente a 13 dólares. La institución privada lucrativa muestra un gasto muy superior en el conjunto. La institución privada sin fines de lucro y con mayor diversificación en sus fuentes de financiamiento muestra un gasto muy por encima del promedio. Las dos instituciones públicas, por el contrario, ejercen un gasto muy por debajo del promedio.

Los hogares en su conjunto son los que hacen las mayores aportaciones financieras a los programas dentro de las instituciones privadas lucrativas. Sin embargo, las instituciones que atienden a población con menor ingreso, deben buscar fuentes alternativas de financiamiento como la autogestión -que les permite funcionar- dado que los hogares de sus pacientes deben dedicar sus ingresos a la propia subsistencia. En sentido estricto, los hogares y empresas también participan en los aportes del gobierno, al pagar impuestos. Sin embargo, en los hogares de bajo ingreso, los gastos de bolsillo para la atención de la farmacodependencia son reducidos.

La importancia del monto según la fuente de financiamiento depende de la institución de que se trate, ya sea privada o pública. De acuerdo con los resultados de este estudio, la mayor parte del presupuesto de la institución 1 proviene de los hogares, en tanto que la mayoría de los recursos de la institución 2 proviene de empresas y de la autogestión. Las instituciones 3 y 4 , por su parte, reciben aportaciones del gobierno.

Hay que resaltar de manera positiva el hecho de que, en las instituciones privadas, exista una mezcla de estrategias de financiamiento, y si bien todas ellas son privadas, su diversidad impide que los gastos se conviertan en una carga financiera para los hogares, lo que sucedería si los recursos tuviesen un solo origen. La diversificación de los aportes resulta, por lo tanto, una estrategia recomendable, ya que las instituciones con una mayor diversidad de fuentes de financiamiento son las que poseen un crecimiento mayor en el monto de sus recursos.

Un aspecto importante es que pareciera inexistente la modalidad de financiamiento por seguro médico privado o prepago. Eso se debe a que la farmacodependencia no ha sido vista como una problemática social prioritaria que debe recibir una parte del gasto en salud de los hogares, para su prevención y manejo. Recientemente, después de haber concluido este trabajo, los niveles de adicción se han elevado en la población joven en forma alarmante, por lo que el manejo financiero de los programas de atención a la farmacodependencia deberá incorporar modalidades de pago que no afecten los presupuestos familiares sino en una parte mínima.

Es importante resaltar que, al igual que los montos de financiamiento, la demanda de servicio mostró una tendencia creciente en el periodo 1990-1993, aunque no tan marcada. En efecto, la demanda de servicios se mantuvo por debajo de la media de incremento del monto financiero, lo cual de alguna manera favoreció un mejor manejo de los recursos.

Los hallazgos de este estudio también manifies$\tan$ que las cuotas establecidas por las instituciones dedicadas a atender a la población abierta, no cubren los gastos de operación de este tipo de programas; empero, deberían ajustarse a la dinámica económica actual, al ingreso económico de los usuarios (que cada día se ve más afectado), al crecimiento de la demanda $\mathrm{y}$ a las necesidades propias del programa.

En cuanto a la asignación de recursos por tipo de programa, el hecho de que no existan mecanismos de asignación explícitos ni información disponible sobre los montos específicos para actividades de promoción, prevención, curación y rehabilitación, dificulta de manera sustantiva el análisis de los flujos de financiamiento.

Uno de los principios básicos de la eficiencia en medicina es hacer hincapié en la prevención, lo que implica no dar prioridad únicamente a la atención clínica. No obstante, en este estudio se observa que el 
discurso en favor de la prevención dista mucho de la realidad. El análisis de flujos financieros demuestra que la energía de estas instituciones, así como sus recursos materiales y humanos, están dirigidos a la parte clínica y no a la prevención.

Aunque ya se resaltó la desproporción del gasto de las instituciones públicas en relación con el de las instituciones privadas, en lo referente a los montos de gasto per cápita, el problema se agudiza; es decir, mientras que los montos per cápita para las instituciones privadas se colocaron por arriba de la media, los montos para las instituciones públicas se ubicaron muy por debajo. Esto implica que, en materia de gasto y financiamiento en salud, los recursos dirigidos a instituciones públicas resultan muy inferiores a los de las privadas y seguramente insuficientes para responder a las demandas crecientes de la población no asegurada, en materia de drogadicción.

Todo lo anterior pone de manifiesto que, si se quiere contar con flujos financieros saludables, es necesario, en primer lugar, impulsar nuevos mecanismos de financiamiento, promover más la mezcla de financiamiento público-privado, así como desarrollar e implementar mecanismos de asignación financiera en función de los montos económicos necesarios y de los diferentes niveles de demanda. Estas tres acciones, entre otras, podrían constituirse en la base para poder identificar indicadores de financiamiento, de tal manera que quienes se encargan de tomar decisiones cuenten con la información pertinente y actualizada para hacer los cambios y ajustes necesarios en las políticas de financiamiento de los programas estudiados en este trabajo.

\section{Recomendaciones}

Los resultados de esta investigación deben darse a conocer no sólo en las instituciones estudiadas, sino también en otras que, de alguna manera, están involucradas en el financiamiento de programas de apoyo a la rehabilitación de los farmacodependientes en México. Esta tarea podría ser asumida por el Conadic, que tiene un mayor contacto con las instituciones del país involucradas en la materia. De esta manera se podrían crear condiciones para que las instituciones brinden más información y, a su vez, obtengan un mayor beneficio de los resultados generados por este tipo de investigaciones.

Es importante una delimitación clara de las asignaciones financieras en las instituciones dedicadas a atender la farmacodependencia, ya que de esta manera los recursos se podrían optimizar y destinar eficazmente a la satisfacción integral de las necesidades y demandas de la población farmacodependiente. Las instituciones podrían desarrollar estudios que sometan a prueba diferentes modalidades de financiamiento, combinándolas con la producción autogestiva de servicios. En esos estudios podrían participar instituciones públicas o con financiamiento privado.

Hay que resaltar la experiencia de autogestión de la institución 2. En otras instituciones, esta alternativa de financiamiento incrementaría los aportes, contribuiría a elevar la calidad de la atención médica y satisfaría en mayor medida la demanda del usuario. Además, este tipo de fuentes financieras posibilitaría la capacitación del farmacodependiente en el manejo de algún oficio, ya sea manual o técnico, y le abriría oportunidades de inserción en el mercado de trabajo.

Es importante hacer énfasis en la formación continua de promotores de salud, seleccionados de entre los grupos de farmacodependientes rehabilitados. Ello implicaría menor inversión en la contratación de personal o mano de obra a menor costo. Con base en los ejemplos antes expuestos -que en México no se presentan en forma aislada-, sugerimos realizar campañas de prevención entre la población infantil, ya que ello contribuiría, a la vez, a la reducación de los adultos. En este sentido, hay experiencias exitosas en América Latina, específicamente en Venezuela, como el programa de cuidado ambiental Educando al infante para educar al adulto.

En el contexto de las reformas y la descentralización de los servicios de salud, es altamente prioritario que las autoridades locales participen activamente en la elección de mecanismos eficientes para la asignación de recursos, con el fin de evitar problemas financieros. ${ }^{16} \mathrm{La}$ recomendación puntual en este nivel de acción es la generación de bases de información que permitan a quienes toman decisiones replantear, evaluar y ajustar las políticas de financiamiento a las necesidades de salud de la población, pero también al saneamiento administrativo y financiero de los propios sistemas de salud.

Por último, otra propuesta sería que parte del dinero y de las propiedades incautadas por acciones contra el narcotráfico sean utilizados para la prevención y la curación de la farmacodependencia. Con estos fondos se podría pensar en la creación de paquetes de tratamiento, de servicios de información telefónica las 24 horas del día, de centros de trabajo en grupo para formular campañas dirigidas a los consumidores de drogas y de grupos de autoapoyo, entre otras opciones. 


\section{Referencias}

1. Centros de Integración Juvenil. Riesgos macrosociales de la farmacodependencia y red de estrategias de atención en México. En: Plan Rector Institucional a Mediano Plazo 1989-1994. México, D.F.: CIJ (Serie Técnica, vol. 6, Perfil Gestión Institucional), 1989:1.

2. Secretaría de Salud. Programa de Salud 1990-1994. México, D.F.: S.S.A, 1990.

3. Soberón-A cevedo G. El cambio estructural en la salud. IV. El financiamiento de la salud para consolidar el cambio. Salud Publica Mex 1987; 29(2):169-177.

4. A kin J. Financing health services in developing countries:An agenda reform. W ashington, D.C.: W orld Bank, 1987.

5. Centros de Integración Juvenil. U na propuesta integral al fenómeno de la farmacodependencia. México, D.F.: CIJ, 1982.

6. Centros de Integración Juvenil. A portaciones teóricas y prácticas para el conocimiento del farmacodependiente. México, D.F.: CI) (Serie Técnica, vol. 5, Perfil Codependiente), 1984.

7. Cruz C, Alvarez F, Frenk J, Knaul F, Valdés C, Ramírez R. Las cuentas nacionales de salud y el financiamiento. México, D.F.: Fundación Mexicana para la Salud, 1994.

8. Ruiz de Chávez M, Ayala-Cabrera R. Planeación y financiamiento en salud: nuevos esquemas ante la crisis. México, D.F.: Secretaría de Salud/
Gobierno del Estado de N uevo León / O rganización Panamericana de la Salud, 1988.

9. Bobadilla JL, Frenk J, Cowley P, Zurita B, Q uerol J,Villareal E et al. R. El paquete universal de servicios esenciales de salud. México, D.F.: Fundación Mexicana para la Salud (D ocumento para el Análisis y la Convergencia, núm.11), 1994.

10. Lozano R, Murray C, Frenk J, Bobadilla JL, Fernández S. El peso de la enfermedad en México: un doble reto. México, D.F.: Fundación Mexicana para la Salud (Serie Economía y Salud), 1994.

11. Hernández-Peña P, Cruz C, Z urita B, Frenk J, Ramírez R,Alvarez F. Las cuentas nacionales de salud. México, D.F.:Fundación Mexicana para la Salud (O bservatorio de la Salud), 1997: 119-142.

12. Arredondo A. Financial indicators for health care decentralization in developing countries: A framework for analysis. Health and System Science 1998;2(1):345-364.

13. N ordhaus S. Economía. 12a ed. México, D.F.: Mc Graw Hill, 1993: 124-125, 271, 1077-1078.

14. Sistema N acional de Encuestas. Encuesta N acional de Adicciones 1987. México D.F.: Secretaría de Salud / Instituto Mexicano de Psiquiatría, 1988. 15. Sistema N acional de Encuestas. Encuesta N acional deA dicciones 1993. México. D.F.: Secretaría de Salud / Instituto Mexicano de Psiquiatría, 1994. 16. Arredondo A. Indicadores de eficiencia para la asignación de recursos financieros en salud. Rev Med Chil 1999;127:856-961. 